\title{
A note on type 2 degenerate poly-Frobenius-Euler polynomials
}

\author{
Waseem A. Khan
}

Department of Mathematics and Natural Sciences, Prince Mohammad Bin Fahd University, P.O Box 1664, Al Khobar 31952, Saudi Arabia

E-mail: wkhan1@pmu.edu.sa

\begin{abstract}
In this paper, we construct the degenerate poly-Frobenius-Genocchi polynomials, called the type 2 degenerate poly-Frobenius-Euler polynomials, by means of polyexponential function. We derive explicit expressions and some identities of those polynomials. In the last section, we introduce type 2 degenerate unipoly-FrobeniusGenocchi polynomials by means of unipoly function and derive explicit multifarious properties.
\end{abstract}

Keywords: polylogarithm function, Frobenius-Genocchi polynomials, degenerate poly-Frobenius-Euler polynomials.

2010 Mathematics Subject Classification: 05A19, 11B68, 11B83, 11B95.

\section{Introduction}

Special polynomials and their generating functions have important roles in many branches of mathematics, probability, statistics, mathematical physics, and also engineering. Since polynomials are suitable for applying well-known operations such as derivative and integral, polynomials are very useful to study real-world problems in the aforementioned areas. For instance, generating functions for special polynomials with their congruence properties, recurrence relations, computational formulae, and symmetric sum involving these polynomials have been many authors in recent years (see $[1-25])$.

Recently, Kim and his research team (see [13-19]) have studied the degenerate versions of special numbers and polynomials actively. This idea provides a powerfool tool in order to define special numbers and polynomials of their degenerate versions. We can say that the notion of degenerate version from a special class of polynomials because of their great applicability. The most important of application of these polynomials are in theory of finite differences, analytic number theory, applications in classical analysis and statistics. Despite the applicability of special functions in classical analysis and statistics, they also arise in communications systems, quantum mechanics, nonlinear wave propagation, electric circuit theory. electromagnetic theory, etc.

Throughout this presentation, we use the following standard notions $\mathbb{N}=\{1,2, \cdots\}$, $\mathbb{N}_{0}=\{0,1,2, \cdots\}=\mathrm{N} \cup\{0\}, \mathbb{Z}^{-}=\{-1,-2, \cdots\}$. Also as usual $\mathbb{Z}$ denotes the set of integers, $\mathbb{R}$ denotes the set of real numbers and $\mathbb{C}$ denotes the set of complex numbers.

The classical Bernoulli $B_{n}(x)$, Euler $E_{n}(x)$ and Genocchi $G_{n}(x)$ polynomial are defined by means of the following generating function as follows

$$
\frac{t}{e^{t}-1} e^{x t}=\sum_{n=0}^{\infty} B_{n}(x) \frac{t^{n}}{n !},|t|<2 \pi, \frac{2}{e^{t}+1} e^{x t}=\sum_{n=0}^{\infty} E_{n}(x) \frac{t^{n}}{n !},|t|<\pi,
$$

and

$$
\frac{2 t}{e^{t}+1} e^{x t}=\sum_{n=0}^{\infty} G_{n}(x) \frac{t^{n}}{n !},|t|<\pi,(\text { see }[6,7,19,20,23])
$$


respectively.

For $u \in \mathbb{C}$ with $u \neq 1$, the classical Frobenius-Euler polynomials $H_{n}^{(\alpha)}(x ; u)$ of order $\alpha$ are defined by means of the following generating function

$$
\left(\frac{1-u}{e^{t}-u}\right)^{\alpha} e^{x t}=\sum_{n=0}^{\infty} H_{n}^{(\alpha)}(x ; u) \frac{t^{n}}{n !},(\text { see }[1,5,11,12]) .
$$

In the special case when $x=0, H_{n}^{(\alpha)}(u)=H_{n}^{(\alpha)}(0 ; u)$ are called $n^{\text {th }}$ FrobeniusEuler numbers of order $\alpha$. For $\alpha=1$ into $(1.2), H_{n}^{(1)}(x, u)=H_{n}(x, u)$, are called the Frobenius-Euler polynomials and $H_{n}^{(\alpha)}(0 ; u)=h_{n}^{(\alpha)}(u)$, are called the Frobenius-Euler numbers of order $\alpha$. Substituting $u=-1$ into $(1.2), H_{n}(x ;-1)=E_{n}(x)$, are called the Euler polynomials, (see [8, 22, 24, 25]). by

In (2017), Kurt [10] introduced the poly-Frobenius-Euler polynomials are given

$$
\frac{(1-u) \operatorname{Li}_{k}\left(1-e^{-t}\right)}{t\left(e^{t}-u\right)} e^{x t}=\sum_{n=0}^{\infty} H_{n}^{(k)}(x ; u) \frac{t^{n}}{n !} .
$$

In the case when $x=0, H_{n}^{(k)}(u)=H_{n}^{(k)}(0 ; u)$ are called the poly-FrobeniusEuler numbers. by

For any non-zero $\lambda \in \mathbb{R}$ (or $\mathbb{C}$ ), the degenerate exponential function is defined

$$
e_{\lambda}^{x}(t)=(1+\lambda t)^{\frac{x}{\lambda}}, e_{\lambda}(t)=(1+\lambda t)^{\frac{1}{\lambda}},(\text { see }[13,18,19]) .
$$

By binomial expansion, we get

$$
e_{\lambda}^{x}(t)=\sum_{n=0}^{\infty}(x)_{n, \lambda} \frac{t^{n}}{n !},(\text { see }[14,17]),
$$

where $(x)_{0, \lambda}=1,(x)_{n, \lambda}=(x-\lambda)(x-2 \lambda) \cdots(x-(n-1) \lambda),(n \geq 1)$.

Note that

$$
\lim _{\lambda \longrightarrow 0} e_{\lambda}^{x}(t)=\sum_{n=0}^{\infty} x^{n} \frac{t^{n}}{n !}=e^{x t} .
$$

The degenerate Bernoulli and degenerate Euler polynomials are respectively, given by Carlitz $[2,3]$ as follows:

$$
\frac{z}{e_{\lambda}(z)-1} e_{\lambda}^{x}(z)=\sum_{j=0}^{\infty} B_{j, \lambda}(x) \frac{z^{j}}{j !}, \frac{2}{e_{\lambda}(z)+1} e_{\lambda}^{x}(z)=\sum_{j=0}^{\infty} E_{j, \lambda}(x) \frac{z^{j}}{j !} .
$$

In the case when $x=0, B_{j, \lambda}=B_{j, \lambda}(0)$ are called the degenerate Bernoulli numbers and $x=0, E_{j, \lambda}=E_{j, \lambda}(0)$ are called the degenerate Euler numbers.

Note that

$$
\lim _{\lambda \longrightarrow 0} \beta_{n}(x ; \lambda)=B_{n}(x), \lim _{\lambda \longrightarrow 0} E_{n}(x ; \lambda)=E_{n}(x) .
$$

Kim et al. [15] introduced the degenerate Frobenius-Euler polynomials are defined by means of the generating function as follows

$$
\frac{1-u}{(1+\lambda t)^{\frac{1}{\lambda}}-u}(1+\lambda t)^{\frac{x}{\lambda}}=\sum_{n=0}^{\infty} h_{n, \lambda}(x \mid u) \frac{t^{n}}{n !},
$$


At the value $x=0, h_{n, \lambda}(u)=h_{n, \lambda}(0 \mid u)$ are called the degenerate FrobeniusEuler numbers.

It is readily seen that

$$
\lim _{\lambda \longrightarrow 0} h_{n, \lambda}(x \mid u)=H_{n}(x \mid u),(n \geq 0) .
$$

Kim-Kim [13] introduced the polyexponential function, as an inverse to the polylogarithm function to be

$$
\operatorname{Ei}_{k}(x)=\sum_{n=1}^{\infty} \frac{x^{n}}{(n-1) ! n^{k}},(k \in \mathbb{Z}) .
$$

For $k=1,(1.8)$ gives

$$
\operatorname{Ei}_{1}(x)=\sum_{n=1}^{\infty} \frac{x^{n}}{n !}=e^{x}-1
$$

For $k \in \mathbb{Z}$, Kim et al. [18] introduced the degenerate poly-Bernoulli polynomials defined by

$$
\frac{\operatorname{Ei}_{k}(\log (1+t))}{e_{\lambda}(t)-1} e_{\lambda}^{x}(t)=\sum_{n=0}^{\infty} \beta_{n, \lambda}^{(k)}(x) \frac{t^{n}}{n !}
$$
numbers.

In the special case $x=0, \beta_{n, \lambda}^{(k)}=\beta_{n, \lambda}^{(k)}(0)$ are called the degenerate poly-Bernoulli

It is well known that the Stirling numbers of the first kind are defined by

$$
(x)_{n}=\sum_{l=0}^{n} S_{1}(n, l) x^{l},(\text { see }[24,25]),
$$

where $(x)_{0}=1$, and $(x)_{n}=x(x-1) \cdots(x-n+1),(n \geq 1)$. From (1.11), it is easily to see that

$$
\frac{1}{k !}(\log (1+t))^{k}=\sum_{n=k}^{\infty} S_{1}(n, k) \frac{t^{n}}{n !},(k \geq 0),(\text { see }[13,18]) .
$$

In the inverse expression to (1.12), the Stirling numbers of the second kind are defined by

$$
x^{n}=\sum_{l=0}^{n} S_{2}(n, l)(x)_{l},(\text { see }[10,12,22]) .
$$

From (1.13), it is easily to see that

$$
\frac{1}{k !}\left(e^{t}-1\right)^{k}=\sum_{n=l}^{\infty} S_{2}(n, l) \frac{t^{n}}{n !},(\text { see }[22,24,25]) .
$$

For $n \geq 0$, the degenerate Stirling numbers of the second kind $[7,8,17]$ are defined by

$$
\frac{1}{n !}\left(e_{\lambda}(t)-1\right)^{n}=\sum_{l=n}^{\infty} S_{2, \lambda}(l, n) \frac{t^{l}}{l !},(n \geq 0) .
$$

In this paper, we construct the degenerate poly-Frobenius-Euler polynomials and numbers, called the type 2 oly-Frobenius-Euler polynomials and numbers by using the polyexponential function and derive several properties on the degenerate poly-Frobenius-Euler polynomials and numbers. In the final section, we define type 2 unipoly-Frobenius-Euler polynomials by means of unipoly function and derive explicit 
expressions of those polynomials.

\section{Type 2 degenerate poly-Frobenius-Euler polynomials}

Let $\lambda, u \in \mathbb{C}$ with $u \neq 1$ and $k \in \mathbb{Z}$, by using the polyexponential function, we consider the type 2 degenerate poly-Frobenius-Euler polynomials are defined by means of the following generating function

$$
\frac{\operatorname{Ei}_{k}(\log (1+(1-u) t))}{t\left(e_{\lambda}(t)-u\right)} e_{\lambda}^{x}(t)=\sum_{n=0}^{\infty} H_{n, \lambda}^{(k)}(x ; u) \frac{t^{n}}{n !} .
$$

In the special case, $x=0, H_{n, \lambda}^{(k)}(u)=H_{n, \lambda}^{(k)}(0 ; u)$ are called the type 2 degenerate poly-Frobenius-Euler numbers.

For $k=1$ in $(2.1)$, we get

$$
\frac{1-u}{e_{\lambda}(t)-u} e_{\lambda}^{x}(t)=\sum_{n=0}^{\infty} h_{n, \lambda}(x ; u) \frac{t^{n}}{n !},(\text { see []) }
$$

where $h_{n, \lambda}(x ; u)$ are called the degenerate Frobenius-Euler polynomials.

Obviously

$$
\begin{gathered}
\lim _{\lambda \longrightarrow 0}\left(\frac{\operatorname{Ei}_{k}(\log (1+(1-u) t))}{t\left(e_{\lambda}(t)-u\right)}\right) e_{\lambda}^{x}(t)=\sum_{n=0}^{\infty} \lim _{\lambda \rightarrow 0} H_{n, \lambda}^{(k)}(x ; u) \frac{t^{n}}{n !} \\
=\frac{\operatorname{Ei}_{k}(\log (1+(1-u) t))}{t\left(e^{t}-u\right)} e^{x t}=\sum_{n=0}^{\infty} H_{n}^{(k)}(x ; u) \frac{t^{n}}{n !} .
\end{gathered}
$$

Thus, by (2.1) and (2.2), we have

$$
\lim _{\lambda \longrightarrow 0} H_{n, \lambda}^{(k)}(x ; u)=H_{n}^{(k)}(x ; u),(n \geq 0)
$$

where $H_{n}^{(k)}(x ; u)$ are called the type 2 poly-Frobenius-Euler polynomials, (see [15]).

Theorem 2.1. For $n \geq 0$, we have

$$
\begin{gathered}
\sum_{l=0}^{n}\left(\begin{array}{l}
n \\
l
\end{array}\right) \sum_{m=0}^{l} \frac{1}{(m+1)^{k-1}} S_{1}(l+1, m+1)(x)_{n-l, \lambda} \frac{(1-u)^{l+1}}{l+1} \\
=\sum_{m=0}^{n}\left(\begin{array}{l}
n \\
m
\end{array}\right) H_{n-m, \lambda}^{(k)}(x ; u)(1)_{m, \lambda}-u H_{n, \lambda}^{(k)}(x ; u) .
\end{gathered}
$$

Proof. From (2.1), we have

$$
\begin{gathered}
\frac{\operatorname{Ei}_{k}(\log (1+(1-u) t))}{t} e_{\lambda}^{x}(t)=e_{\lambda}(t) \sum_{n=0}^{\infty} H_{n, \lambda}^{(k)}(x ; u) \frac{t^{n}}{n !}-u \sum_{n=0}^{\infty} H_{n, \lambda}^{(k)}(x ; u) \frac{t^{n}}{n !} \\
=\sum_{m=0}^{\infty}(1)_{m, \lambda} \frac{t^{m}}{m !} \sum_{n=0}^{\infty} H_{n, \lambda}^{(k)}(x ; u) \frac{t^{n}}{n !}-u \sum_{n=0}^{\infty} H_{n, \lambda}^{(k)}(x ; u) \frac{t^{n}}{n !} \\
=\sum_{n=0}^{\infty}\left(\sum_{m=0}^{n}\left(\begin{array}{c}
n \\
m
\end{array}\right) H_{n-m, \lambda}^{(k)}(x ; u)(1)_{m, \lambda}-u H_{n, \lambda}^{(k)}(x ; u)\right) \frac{t^{n}}{n !} .
\end{gathered}
$$

On the other hand,

$$
\frac{\operatorname{Ei}_{k}(\log (1+(1-u) t))}{t} e_{\lambda}^{x}(t)
$$




$$
\begin{aligned}
= & \left(\sum_{n=0}^{\infty}(x)_{n, \lambda} \frac{t^{n}}{n !}\right) \frac{1}{t}\left(\sum_{m=1}^{\infty} \frac{(\log (1+(1-u) t))^{m}}{(m-1) ! m^{k}}\right) \\
= & \left(\sum_{n=0}^{\infty}(x)_{n, \lambda} \frac{t^{n}}{n !}\right) \frac{1}{t}\left(\sum_{m=0}^{\infty} \frac{1}{(m+1)^{k-1}} \sum_{l=m+1}^{\infty} S_{1}(l, m+1) \frac{(1-u)^{l} t^{l}}{l !}\right) \\
= & \left(\sum_{n=0}^{\infty}(x)_{n, \lambda} \frac{t^{n}}{n !}\right)\left(\sum_{l=0}^{\infty} \sum_{m=0}^{l} \frac{1}{(m+1)^{k-1}} S_{1}(l+1, m+1) \frac{(1-u)^{l+1}}{l+1} \frac{t^{l}}{l !}\right) \\
\text { L.H.S } & =\sum_{n=0}^{\infty}\left(\sum_{l=0}^{n}\left(\begin{array}{c}
n \\
l
\end{array}\right) \sum_{m=0}^{l} \frac{1}{(m+1)^{k-1}} S_{1}(l+1, m+1)(x)_{n-l, \lambda} \frac{(1-u)^{l+1}}{l+1}\right) \frac{t^{n}}{n !} .
\end{aligned}
$$

Comparing the coefficients of $\frac{t^{n}}{n !}$ on both sides of equation (2.4) and (2.5), we obtain the following theorem.

Theorem 2.2. For $n \geq 0$, we have

$$
H_{n, \lambda}^{(k)}(x ; u)=\sum_{m=0}^{n}\left(\begin{array}{l}
n \\
m
\end{array}\right) H_{n-m, \lambda}^{(k)}(u)(x)_{m, \lambda} .
$$

Proof. From (2.1), we have

$$
\begin{gathered}
\sum_{n=0}^{\infty} H_{n, \lambda}^{(k)}(x ; u) \frac{t^{n}}{n !}=\left(\frac{\operatorname{Ei}_{k}(\log (1+(1-u) t))}{t\left(e_{\lambda}(t)-u\right)}\right) e_{\lambda}^{x}(t) \\
=\sum_{n=0}^{\infty} H_{n, \lambda}^{(k)}(u) \frac{t^{n}}{n !} \sum_{m=0}^{\infty}(x)_{m, \lambda} \frac{t^{m}}{m !} \\
\text { L.H.S }=\sum_{n=0}^{\infty} \sum_{m=0}^{n}\left(\begin{array}{c}
n \\
m
\end{array}\right) H_{n-m, \lambda}^{(k)}(u)(x)_{m, \lambda} \frac{t^{n}}{n !} .
\end{gathered}
$$

Therefore, by (2.1) and (2.6), we require at the desired result.

Theorem 2.3. For $k \in \mathbb{Z}$ and $n \geq 0$, we have

$$
H_{n, \lambda}^{(k)}(x ; u)=\sum_{l=0}^{n}\left(\begin{array}{l}
n \\
l
\end{array}\right) \sum_{m=0}^{l} \frac{1}{(m+1)^{k}} \frac{S_{1}(l+1, m+1)(1-u)^{l}}{l+1} H_{n-l, \lambda}(x ; u) .
$$

Proof. By using equations (1.7), (1.12) and (2.1), we have

$$
\begin{gathered}
\sum_{n=0}^{\infty} H_{n, \lambda}^{(k)}(x ; u) \frac{t^{n}}{n !}=\left(\frac{\operatorname{Ei}_{k}(\log (1+(1-u) t))}{t\left(e_{\lambda}(t)-u\right)}\right) e_{\lambda}^{x}(t) \\
=\frac{e_{\lambda}^{x}(t)}{t\left(e_{\lambda}(t)-u\right)} \sum_{m=1}^{\infty} \frac{(\log (1+(1-u) t))^{m}}{(m-1) ! m^{k}} \\
=\frac{e_{\lambda}^{x}(t)}{t\left(e_{\lambda}(t)-u\right)} \sum_{m=0}^{\infty} \frac{(\log (1+(1-u) t))^{m+1}}{m !(m+1)^{k}} \\
=\frac{e_{\lambda}^{x}(t)}{t\left(e_{\lambda}(t)-u\right)} \sum_{m=0}^{\infty} \frac{1}{(m+1)^{k}} \sum_{n=m+1}^{\infty} S_{1}(n, m+1) \frac{((1-u) t)^{n}}{n !} \\
=\frac{1-u}{e_{\lambda}(t)-u} e_{\lambda}^{x}(t) \sum_{m=0}^{\infty} \frac{1}{(m+1)^{k}} \sum_{m=n}^{\infty} \frac{S_{1}(n+1, m+1)(1-u)^{n}}{n+1} \frac{t^{n}}{n !}
\end{gathered}
$$




$$
\begin{gathered}
=\sum_{n=0}^{\infty} H_{n, \lambda}(x ; u) \frac{t^{n}}{n !} \sum_{l=0}^{\infty} \sum_{m=0}^{l} \frac{1}{(m+1)^{k}} \frac{S_{1}(l+1, m+1)(1-u)^{l}}{l+1} \frac{t^{l}}{l !} \\
\text { L.H.S }=\sum_{n=0}^{\infty}\left(\sum_{l=0}^{n}\left(\begin{array}{c}
n \\
l
\end{array}\right) \sum_{m=0}^{l} \frac{1}{(m+1)^{k}} \frac{S_{1}(l+1, m+1)(1-u)^{l}}{l+1} H_{n-l, \lambda}(x ; u)\right) \frac{t^{n}}{n !} .
\end{gathered}
$$

By comparing the coefficients of $\frac{t^{n}}{n !}$, we complete the proof.

Corollary 2.1. For $k \in \mathbb{Z}$ and $n \geq 0$, we have

$$
H_{n, \lambda}^{(k)}(u)=\sum_{l=0}^{n}\left(\begin{array}{l}
n \\
l
\end{array}\right) \sum_{m=0}^{l} \frac{1}{(m+1)^{k}} \frac{S_{1}(l+1, m+1)(1-u)^{l}}{l+1} H_{n-l, \lambda}(u) .
$$

Corollary 2.2. For $n \geq 0$, we have

$$
H_{n, \lambda}(x ; u)=\sum_{l=0}^{n}\left(\begin{array}{l}
n \\
l
\end{array}\right) \sum_{m=0}^{l} \frac{S_{1}(l+1, m+1)(1-u)^{l}}{l+1} H_{n-l, \lambda}(x ; u) .
$$

Corollary 2.3. For $n \geq 0$, we have

$$
E_{n, \lambda}(x)=\sum_{l=0}^{n}\left(\begin{array}{c}
n \\
l
\end{array}\right) \sum_{m=0}^{l} \frac{S_{1}(l+1, m+1) 2^{l}}{l+1} E_{n-l, \lambda}(x) .
$$

In particular,

$$
\sum_{l=0}^{n}\left(\begin{array}{l}
n \\
l
\end{array}\right) \sum_{m=0}^{l} \frac{S_{1}(l+1, m+1) 2^{l}}{l+1} E_{n-l, \lambda}(x)=0 .
$$

It is well-known from $([16,21])$ that

$$
\left(\frac{t}{\log (1+t)}\right)^{r}(1+t)^{x-1}=\sum_{n=0}^{\infty} B_{n}^{(n-r+1)}(x) \frac{t^{n}}{n !},(r \in \mathbb{C}),
$$

where $B_{n}^{(r)}(x)$ are called the higher-order Bernoulli polynomials which are given by the generating function

$$
\left(\frac{t}{e^{t}-1}\right)^{r} e^{x t}=\sum_{n=0}^{\infty} B_{n}^{(r)}(x) \frac{t^{n}}{n !}
$$

Theorem 2.4. For $n \geq 0$, we have

$$
H_{n, \lambda}^{(2)}(u)=\sum_{l=0}^{l}\left(\begin{array}{l}
n \\
l
\end{array}\right) \frac{(1-u)^{l} B_{l}^{l}}{l+1} H_{n-l, \lambda}(u) .
$$

Proof. Using (1.8), we first consider the following expression

$$
\begin{gathered}
\frac{d}{d x} \operatorname{Ei}_{k}(\log (1+(1-u) x))=\frac{d}{d x} \sum_{n=1}^{\infty} \frac{(\log (1+(1-u) x))^{n}}{(n+1) ! n^{k}} \\
=\frac{1-u}{(1+(1-u) x) \log (1+(1-u) x)} \sum_{n=1}^{\infty} \frac{(\log (1+(1-u) x))^{n}}{(n+1) ! n^{k-1}} \\
=\frac{1-u}{(1+(1-u) x) \log (1+(1-u) x)} E i_{k-1}(\log (1+(1-u) x) .
\end{gathered}
$$

From (2.9), $k \geq 1$, we have 


$$
\begin{gathered}
\sum_{n=0}^{\infty} H_{n, \lambda}^{(k)}(u) \frac{x^{n}}{n !}=\frac{(1-u)^{k-1}}{x\left(e_{\lambda}(x)-u\right)} \int_{0}^{x} \frac{1}{(1+(1-u) t) \log (1+(1-u) t)} \\
\times \int_{0}^{t} \underbrace{\frac{1}{(1+(1-u) t) \log (1+(1-u) t)} \cdots \int_{0}^{t} \frac{t}{(1+(1-u) t) \log (1+(1-u) t)} d t d t \ldots d t .}_{k-2-\text { times }}
\end{gathered}
$$

Hence, we require

$$
\begin{aligned}
\sum_{n=0}^{\infty} H_{n, \lambda}^{(2)}(u) \frac{x^{n}}{n !}= & \frac{(1-u)}{x\left(e_{\lambda}(x)-u\right)} \int_{0}^{x} \frac{(1-u) t}{(1+(1-u) t) \log (1+(1-u) t)} \\
= & \frac{(1-u)}{x\left(e_{\lambda}(x)-u\right)} \int_{0}^{x} \sum_{n=0}^{\infty}(1-u)^{n} B_{n}^{n} \frac{t^{n}}{n !} d t \\
& =\frac{(1-u) x}{x\left(e_{\lambda}(x)-u\right)} \sum_{n=0}^{\infty} \frac{(1-u)^{n} B_{n}^{n}}{n+1} \frac{x^{n}}{n !} \\
= & \left(\sum_{n=0}^{\infty} H_{n, \lambda}(u) \frac{x^{n}}{n !}\right)\left(\sum_{n=0}^{\infty} \frac{(1-u)^{n} B_{n}^{n}}{n+1} \frac{x^{n}}{n !}\right) \\
\text { L.H.S } & =\sum_{n=0}^{\infty}\left(\sum_{l=0}^{l}\left(\begin{array}{l}
n \\
l
\end{array}\right) \frac{\left.(1-u)^{l} B_{l}^{l} H_{n-l, \lambda}(u)\right) \frac{x^{n}}{n !} .}{l+1}\right.
\end{aligned}
$$
proof.

By (2.10) and (2.11), we require at the desired result. Thus, we complete the

Theorem 2.5. Let $k \geq 1$ and $m \in \mathbb{N} \bigcup\{0\}, s \in \mathbb{C}$, we have

$$
\chi_{k, u, \nu}(-m)=(1-u)^{-m-1}(-1)^{m} H_{m, \nu}^{(k)}(u) .
$$

Proof. Let $k \geq 1$, be an integer. For $s \in \mathbb{C}$, we define the function $\chi_{k, \nu}(s)$ as

$$
\chi_{k, \nu}(s)=\frac{1}{\Gamma(s)} \int_{0}^{\infty} \frac{z^{s-1}}{z\left(e_{\nu}(z)-u\right)} \operatorname{Ei}_{k}(\log (1+(1-u) z)) d z .
$$

In view of calculation above that $\chi_{k, \nu}(s)$ is holomorphic function for $\Re(s)>0$ because of the comparison test as $\operatorname{Ei}_{k}(\log (1+(1-u) z)) \leq \operatorname{E} 1_{k}(\log (1+(1-u) z))$ with the assumption $(1-u) t \geq 0$. From (2.11), we note that

$$
\begin{gathered}
\chi_{k, \nu}(s)=\frac{(1-u)^{s-1}}{\Gamma(s)} \int_{0}^{\infty} \frac{z^{s-1}}{z\left(e_{\nu}(z)-u\right)} \operatorname{Ei}_{k}(\log (1+(1-u) z)) d z \\
=\frac{(1-u)^{s-1}}{\Gamma(s)} \int_{0}^{1} \frac{z^{s-1}}{z\left(e_{\nu}(z)-u\right)} \operatorname{Ei}_{k}(\log (1+(1-u) z)) d z \\
+\frac{(1-u)^{s-1}}{\Gamma(s)} \int_{1}^{\infty} \frac{z^{s-1}}{z\left(e_{\nu}(z)-u\right)} \operatorname{Ei}_{k}(\log (1+(1-u) z)) d z
\end{gathered}
$$

The second integral converges absolutely for any $s \in \mathbb{C}$ and hence, the second term on the right hand side vanishes at non-positive integers. That is,

$\lim _{s \rightarrow-m}\left|\frac{(1-u)^{s-1}}{\Gamma(s)} \int_{1}^{\infty} \frac{z}{z\left(e_{\nu}(z)-u\right)} \operatorname{Ei}_{k}(\log (1+(1-u) z)) d z\right| \leq \frac{(1-u)^{-m-1}}{\Gamma(-m)} M=0$,

since

$$
\Gamma(s) \Gamma(1-s)=\frac{\pi}{\sin (\pi s)}
$$


On the other hand, for $\Re(s)>0$, the first integral in (2.13) can be written as

$$
\begin{gathered}
\frac{(1-u)^{s-1}}{\Gamma(s)} \int_{0}^{1} \frac{z^{s-1}}{z\left(e_{\nu}(z)-u\right)} \operatorname{Ei}_{k}(\log (1+(1-u) z)) d z \\
=\frac{(1-u)^{s-1}}{\Gamma(s)} \sum_{n=0}^{\infty} \frac{H_{n, \lambda}^{(k)}(u)}{n !} \int_{0}^{1} z^{n+s-1} d z \\
=\frac{(1-u)^{s-1}}{\Gamma(s)} \sum_{n=0}^{\infty} \frac{H_{n, \lambda}^{(k)}(u)}{n !} \frac{1}{n+s} .
\end{gathered}
$$

which defines an entire function of $s$. Thus, we may include that $\chi_{k, \nu}(s)$ can be continued to an entire function of $s$. Further, from (2.14) and (2.15), we obtain

$$
\begin{gathered}
\chi_{k, \nu, u}(-m)=\lim _{s \rightarrow-m} \frac{(1-u)^{s-1}}{\Gamma(s)} \int_{0}^{1} \frac{z^{s-1}}{z\left(e_{\nu}(z)-u\right)} \operatorname{Ei}_{k}(\log (1+(1-u) z)) d z \\
=\lim _{s \rightarrow-m} \frac{(1-u)^{s-1}}{\Gamma(s)} \sum_{r=0}^{\infty} \frac{H_{r, \nu}^{(k)}}{s+r} \frac{1}{r !} \\
=\cdots+0+\cdots+0+\lim _{s \rightarrow-m} \frac{(1-u)^{s-1}}{\Gamma(s)} \frac{1}{s+m} \frac{H_{m, \nu}^{(k)}(u)}{m !}+0+0+\cdots \\
=\lim _{s \rightarrow-m} \frac{(1-u)^{s-1}}{\Gamma(s)} \frac{\Gamma(1-s) \sin \pi s}{\pi} \frac{H_{m, \nu}^{(k)}(u)}{m !}=(1-u)^{-m-1} \Gamma(1+m) \cos (\pi m) \frac{H_{m, \nu}^{(k)}}{m !} \\
=(1-u)^{-m-1}(-1)^{m} H_{m, \nu}^{(k)}(u) .
\end{gathered}
$$

Thus, we complete the proof of this theorem.

Theorem 2.6. For $n \geq 0$, we have

$$
H_{n, \lambda}^{(k)}(x+y ; u)=\sum_{m=0}^{n}\left(\begin{array}{c}
n \\
m
\end{array}\right) H_{n-m, \lambda}^{(k)}(x ; u)(y)_{m, \lambda} .
$$

Proof. From (2.1), we have

$$
\begin{gathered}
\sum_{n=0}^{\infty} H_{n, \lambda}^{(k)}(x+y ; u) \frac{t^{n}}{n !}=\left(\frac{\operatorname{Ei}_{k}(\log (1+(1-u) t))}{t\left(e_{\nu}(t)-u\right)}\right) e_{\lambda}^{x+y}(t) \\
=\left(\sum_{n=0}^{\infty} H_{n, \lambda}^{(k)}(x ; u) \frac{t^{n}}{n !}\right)\left(\sum_{m=0}^{\infty}(y)_{m, \lambda} \frac{t^{m}}{m !}\right) \\
\text { L.H.S }=\sum_{n=0}^{\infty}\left(\sum_{m=0}^{n}\left(\begin{array}{c}
n \\
m
\end{array}\right) H_{n-m, \lambda}^{(k)}(x ; u)(y)_{m, \lambda}\right) \frac{t^{n}}{n !} .
\end{gathered}
$$

Comparing the coefficients on both sides, we get the result.

Theorem 2.7. For $n \geq 0$, we have

$$
H_{n, \lambda}^{(k)}(x+1 ; u)=\sum_{m=0}^{n}\left(\begin{array}{c}
n \\
m
\end{array}\right) H_{n-m, \lambda}^{(k)}(x ; u)(1)_{m, \lambda} .
$$


Proof. By (2.1), we observe that

$$
\begin{gathered}
\sum_{n=0}^{\infty}\left[H_{n, \lambda}^{(k)}(x+1 ; u)-H_{n, \lambda}^{(k)}(x ; u)\right] \frac{t^{n}}{n !}=\left(\frac{\operatorname{Ei}_{k}(\log (1+(1-u) t))}{t\left(e_{\nu}(t)-u\right)}\right) e_{\lambda}^{x}(t)\left[e_{\lambda}(t)-1\right] \\
\text { L.H.S }=\sum_{n=0}^{\infty} \sum_{m=0}^{n}\left(\begin{array}{c}
n \\
m
\end{array}\right) H_{n-m, \lambda}^{(k)}(x ; u)(1)_{m, \lambda} \frac{t^{n}}{n !}-\sum_{n=0}^{\infty} H_{n, \lambda}^{(k)}(x ; u) \frac{t^{n}}{n !}
\end{gathered}
$$

Comparing the coefficients of $t^{n}$ on both sides, we get the .

Theorem 2.8. For $n \geq 0$, we have

$$
H_{n, \lambda}^{(k)}(x ; u)=\sum_{m=0}^{n} \sum_{q=0}^{m}\left(\begin{array}{l}
n \\
m
\end{array}\right)(x)_{q} S_{\lambda}^{(2)}(m, q) H_{n-m, \lambda}^{(k)}(u) .
$$

Proof. From (2.1), we have

$$
\begin{gathered}
\sum_{n=0}^{\infty} H_{n, \lambda}^{(k)}(x ; u) \frac{t^{n}}{n !}=\left(\frac{\operatorname{Ei}_{k}(\log (1+(1-u) t))}{t\left(e_{\nu}(t)-u\right)}\right) e_{\lambda}^{x}(t) \\
=\left(\frac{\operatorname{Ei}_{k}(\log (1+(1-u) t))}{t\left(e_{\nu}(t)-u\right)}\right)\left[e_{\lambda}(t)-1+1\right]^{x} \\
=\left(\frac{\operatorname{Ei}_{k}(\log (1+(1-u) t))}{t\left(e_{\nu}(t)-u\right)}\right)\left(\sum_{q=0}^{\infty}(x)_{q} \sum_{l=q}^{\infty} S_{\lambda}^{(2)}(l, q) \frac{t^{l}}{l !}\right) \\
\text { L.H.S }=\sum_{n=0}^{\infty}\left(\sum_{m=0}^{n} \sum_{q=0}^{m}\left(\begin{array}{c}
n \\
m
\end{array}\right)(x)_{q} S_{\lambda}^{(2)}(m, q) H_{n-m, \lambda}^{(k)}(u)\right) \frac{t^{n}}{n !} .
\end{gathered}
$$

By comparing the coefficients of $t^{n}$ on both sides, we get the result.

Theorem 2.9. For $n \geq 0$, we have

$$
H_{n, \lambda}^{(k)}(x+\alpha \mid u)=\sum_{l=0}^{n} \sum_{m=0}^{l}\left(\begin{array}{c}
n \\
l
\end{array}\right) x^{m} m ! S_{2, \lambda}(l+\alpha, m+\alpha) H_{n-l, \lambda}^{(k)}(u) .
$$

Proof. Replacing $x$ by $x+\alpha$ in (2.1), we have

$$
\begin{aligned}
& \sum_{n=0}^{\infty} H_{n, \lambda}^{(k)}(x+\alpha ; u) \frac{t^{n}}{n !}=\left(\frac{\operatorname{Ei}_{k}(\log (1+(1-u) t))}{t\left(e_{\nu}(t)-u\right)}\right) e_{\lambda}^{x+\alpha}(t) \\
= & \left(\frac{\operatorname{Ei}_{k}(\log (1+(1-u) t))}{t\left(e_{\nu}(t)-u\right)}\right) e_{\lambda}^{\alpha}(t)\left(\sum_{m=0}^{\infty} x^{m}\left(e_{\lambda}(t)-1\right)^{m}\right) \\
= & \left(\frac{\operatorname{Ei}_{k}(\log (1+(1-u) t))}{t\left(e_{\nu}(t)-u\right)}\right)\left(e_{\lambda}^{\alpha}(t) \sum_{m=0}^{\infty} x^{m} m ! \frac{\left(e_{\lambda}(t)-1\right)^{m}}{m !}\right) \\
= & \left(\frac{\operatorname{Ei}_{k}(\log (1+(1-u) t))}{t\left(e_{\nu}(t)-u\right)}\right)\left(e_{\lambda}^{\alpha}(t) \sum_{m=0}^{\infty} x^{m} m ! \sum_{l=m}^{\infty} S_{2, \lambda}(l, m) \frac{t^{l}}{l !}\right) \\
= & \sum_{n=0}^{\infty} H_{n, \lambda}^{(k)}(u) \frac{t^{n}}{n !}\left(\sum_{l=0}^{\infty} \sum_{m=0}^{l} x^{m} m ! S_{2, \lambda}(l+\alpha, m+\alpha) \frac{t^{l}}{l !}\right) \\
\text { L.H.S }= & \sum_{n=0}^{\infty}\left(\sum_{l=0}^{n} \sum_{m=0}^{l}\left(\begin{array}{c}
n \\
l
\end{array}\right) x^{m} m ! S_{2, \lambda}(l+\alpha, m+\alpha) H_{n-l, \lambda}^{(k)}(u)\right) \frac{t^{n}}{n !} .
\end{aligned}
$$

Therefore, by (2.1) and (2.20), we obtain the result. 


\section{Type 2 degenerate unipoly-Frobenius-Euler polynomials}

Let $p$ be any arithmetic function which is a real or complex valued function defined on the set of positive integers $\mathbb{N}$. Kim-Kim [13] defined the unipoly function attached to polynomials $p(x)$ by

$$
u_{k}(x \mid p)=\sum_{n=1}^{\infty} \frac{p(n)}{n^{k}} x^{n},(k \in \mathbb{Z}) .
$$

Moreover,

$$
u_{k}(x \mid 1)=\sum_{n=1}^{\infty} \frac{x^{n}}{n^{k}}=\operatorname{Li}_{k}(x),(\text { see }[4,9]),
$$

is the ordinary polylogaritm function.

By using (3.1), we define the type 2 degenerate unipoly-Frobenius-Euler polynomials by

$$
\frac{u_{k}(\log (1+(1-u) t) \mid p)}{t\left(e_{\nu}(t)-u\right)} e_{\lambda}^{x}(t)=\sum_{n=0}^{\infty} H_{n, \lambda, p}^{(k)}(x ; u) \frac{t^{n}}{n !} .
$$

In the case when $x=0, H_{n, \lambda, p}^{(k)}(u)=H_{n, \lambda, p}^{(k)}(0 ; u)$ are called the type 2 degenerate unipoly-Frobenius-Euler numbers. Let us take $p(n)=\frac{1}{\Gamma(n)}$. Then, we have

$$
\begin{gathered}
\sum_{n=0}^{\infty} H_{n, \lambda, \frac{1}{\Gamma}}^{(k)}(x ; u) \frac{t^{n}}{n !}=\frac{u_{k}\left(\log (1+(1-u) t) \mid \frac{1}{\Gamma} p\right)}{t\left(e_{\nu}(t)-u\right)} e_{\lambda}^{x}(t) \\
=\frac{1}{t\left(e_{\nu}(t)-u\right)} e_{\lambda}^{x}(t) \sum_{m=1}^{\infty} \frac{(\log (1+(1-u) t))^{m}}{m^{k}(m+1) !} \\
=\frac{\operatorname{Ei}_{\mathrm{k}}(\log (1+(1-u) t))}{e_{\lambda}(t)-u} e_{\lambda}^{x}(t) \\
=\sum_{n=0}^{\infty} H_{n, \lambda}^{(k)}(x ; u) \frac{t^{n}}{n !}
\end{gathered}
$$

Thus, we have

$$
H_{n, \lambda, \frac{1}{\Gamma}}^{(k)}(x ; u)=H_{n, \lambda}^{(k)}(x ; u) .
$$

Theorem 3.1. Let $n \in \mathbb{N}$ and $k \in \mathbb{Z}$. Then we have

$$
H_{n, \lambda, p}^{(k)}(u)=\sum_{l=0}^{n} \sum_{m=0}^{l}\left(\begin{array}{l}
n \\
l
\end{array}\right) \frac{p(m+1)(m+1) !}{(m+1)^{k}} \frac{S_{1}(l+1, m+1)}{l+1}(1-u)^{l} H_{n-l, \lambda}(u) .
$$

In particular,

$$
H_{n, \lambda, \frac{1}{\Gamma}}^{(k)}(u)=\sum_{l=0}^{n} \sum_{m=0}^{l}\left(\begin{array}{l}
n \\
l
\end{array}\right) \frac{m+1}{(m+1)^{k}} \frac{S_{1}(l+1, m+1)}{l+1}(1-u)^{l} H_{n-l, \lambda}(u) .
$$

Proof. From (3.5), we get

$$
\sum_{n=0}^{\infty} H_{n, \lambda, p}^{(k)}(u) \frac{t^{n}}{n !}=\frac{u_{k}(\log (1+(1-u) t) \mid p)}{t\left(e_{\nu}(t)-u\right)}
$$




$$
\begin{gathered}
=\frac{1}{t\left(e_{\nu}(t)-u\right)} \sum_{m=1}^{\infty} \frac{p(m)}{m^{k}}(\log (1+(1-u) t))^{m} \\
=\frac{1}{t\left(e_{\nu}(t)-u\right)} \sum_{m=0}^{\infty} \frac{p(m+1)(m+1) !}{(m+1)^{k}} \sum_{l=m+1}^{\infty} S_{1}(l, m+1) \frac{(1-u)^{l} t^{l}}{l !} \\
=\frac{(1-u) t}{t\left(e_{\nu}(t)-u\right)} \sum_{m=0}^{\infty} \frac{p(m+1)(m+1) !}{(m+1)^{k}} \sum_{m=l}^{\infty} \frac{S_{1}(l+1, m+1)}{l+1}(1-u)^{l} \frac{t^{l}}{l !} \\
=\left(\sum_{n=0}^{\infty} H_{n, \lambda}(u) \frac{t^{n}}{n !}\right)\left(\sum_{m=0}^{\infty} \frac{p(m+1)(m+1) !}{(m+1)^{k}} \sum_{m=l}^{\infty} \frac{S_{1}(l+1, m+1)}{l+1}(1-u)^{l} \frac{t^{l}}{l !}\right) \\
=\sum_{n=0}^{\infty}\left(\sum_{l=0}^{n} \sum_{m=0}^{l}\left(\begin{array}{l}
n \\
l
\end{array}\right) \frac{p(m+1)(m+1) !}{(m+1)^{k}} \frac{S_{1}(l+1, m+1)}{l+1}(1-u)^{l} H_{n-l, \lambda}(u)\right) \frac{t^{n}}{n !} .
\end{gathered}
$$

Therefore, by comparing the coefficients on both sides of (3.8), we obtain the result.

Theorem 3.2. Let $n \geq 0$ and $k \in \mathbb{Z}$. Then we have

$$
H_{n, \lambda, p}^{(k)}(x ; u)=\sum_{m=0}^{n} \sum_{q=0}^{m}\left(\begin{array}{c}
n \\
m
\end{array}\right)(x)_{q} S_{\lambda}^{(2)}(m, q) H_{n-m, \lambda, p}^{(k)}(u) .
$$

Proof. Using (3.3), we observe that

$$
\begin{gathered}
\sum_{n=0}^{\infty} H_{n, \lambda, p}^{(k)}(x ; u) \frac{t^{n}}{n !}=\left(\frac{u_{k}(\log (1+(1-u) t))}{t\left(e_{\nu}(t)-u\right)}\right) e_{\lambda}^{x}(t) \\
=\left(\frac{u_{k}(\log (1+(1-u) t))}{t\left(e_{\nu}(t)-u\right)}\right)\left[e_{\lambda}(t)-1+1\right]^{x} \\
=\left(\frac{u_{k}(\log (1+(1-u) t))}{t\left(e_{\nu}(t)-u\right)}\right)\left(\sum_{q=0}^{\infty}(x)_{q} \sum_{l=q}^{\infty} S_{\lambda}^{(2)}(l, q) \frac{t^{l}}{l !}\right) \\
\text { L.H.S }=\sum_{n=0}^{\infty}\left(\sum_{m=0}^{n} \sum_{q=0}^{m}\left(\begin{array}{c}
n \\
m
\end{array}\right)(x)_{q} S_{\lambda}^{(2)}(m, q) H_{n-m, \lambda, p}^{(k)}(u)\right) \frac{t^{n}}{n !} .
\end{gathered}
$$

By comparing the coefficients of $t^{n}$ on both sides, we get the result.

Theorem 3.3. Let $n \geq 0$ and $k \in \mathbb{Z}$. Then we have

$$
H_{n, \lambda, p}^{(k)}(x ; u)=\sum_{m=0}^{n}\left(\begin{array}{c}
n \\
m
\end{array}\right) H_{n-m, \lambda, p}^{(k)}(u)(x)_{m, \lambda} .
$$

Proof. In order to prove that, we observe that

$$
\begin{gathered}
\sum_{n=0}^{\infty} H_{n, \lambda, p}^{(k)}(x ; u) \frac{t^{n}}{n !}=\frac{u_{k}(\log (1+(1-u) t))}{t\left(e_{\nu}(t)-u\right)} e_{\lambda}^{x}(t) \\
=\left(\sum_{n=0}^{\infty} H_{n, \lambda, p}^{(k)}(u) \frac{t^{n}}{n !}\right)\left(\sum_{m=0}^{\infty}(x)_{m, \lambda} \frac{t^{m}}{m !}\right) \\
\text { L.H.S }=\sum_{n=0}^{\infty}\left(\sum_{m=0}^{n}\left(\begin{array}{c}
n \\
m
\end{array}\right) H_{n-m, \lambda, p}^{(k)}(u)(x)_{m, \lambda}\right) \frac{t^{n}}{n !} .
\end{gathered}
$$


By comparing coefficients on both sides of (3.15), we obtain the result.

\section{Conclusions}

Motivated by the definition of the degenerate poly-Bernoulli polynomials introduced by Kim et al. [18], in the present paper, we have considered a class of new generating function for the degenerate Frobenius-Euler polynomials, called the type 2 degenerate poly-Frobenius-Euler polynomials, by means of the polyexponential function. Then, we have derived some useful relations and properties. We have showed that the type 2 degenerate poly-Frobenius-Euler polynomials equal a linear combination of the degenerate Frobenius-Euler polynomials and Stirlings numbers of the first and second kind. In a special case, we have given a relation between the type 2 degenerate Frobenius-Euler polynomials and Bernoulli polynomials of order $n$. Moreover, inspired by the definition of unipoly-Bernoulli polynomials introduced by Kim-Kim [] we have introduced the type 2 degenerate unipoly-Frobenius-Euler polynomials by means of unipoly function and given multifarious properties including degenerate Stirling numbers of the second kind and degenerate Frobenius-Euler polynomials.

Author Contributions: All authors contributed equally to the manuscript and typed, read, and approved final manuscript.

Conflict of Interest: The authors declare no conflict of interest.

Funding: None.

Acknowledgements: None.

\section{References}

[1] Araci, S.; Acikgoz, M. A note on the Frobenius-Euler numbers and polynomials associated with Bernstein polynomials. Adv. Stud. Contemp. Math. 2012, 22(3), 399-406.

[2] Carlitz, L. Degenerate Stirling, Bernoulli and Eulerian numbers. Utilitas Math. 1979, 15, 51-88.

[3] Carlitz, L. A degenerate Staudt-Clausen theorem, Arch. Math. (Basel) 1956, 7, 28-33.

[4] Eastham, M.S.P. On Polylogarithms. Proc. Glasgow Math. Assoc. 1964, 6, 169-171.

[5] Janson, S, Frobenius numbers and rounding, Reprint (2013), arXiv:1305.3512.

[6] Khan, W.A.; Srivastava, D. On the generalized Apostol type FrobeniusGenocchi polynomials. Filomat. 2019, 33(7), 1967-1977.

[7] Khan, W.A.; Ahmad, M. Partially degenerate poly-Bernoulli polynomials. Adv. Stud. Contemp. Math., 2018, 28(3), 487-496.

[8] Khan, W.A. A new class of degenerate Frobenius-Euler Hermite polynomials. Adv. Stud. Contemp. Math., 2018, 30(4), 567-576.

[9] Kaneko, M. Poly-Bernoulli numbers. J. Théor Nombres Bordeaux. 1997, 9(1), 221-228.

[10] Kurt, B. Poly-Frobenius-Euler polynomials, Proceedings of the International Conferences on Numerical Analysis and Applied Mathematics. Amer, Inst. Phys. Conf. Proc. 2016, 1863, 300019-1-300019-3. 
[11] Kurt, B,; Simsek, Y. On the generalized Apostol type Frobenius Euler polynomials. Advances in Differences equations. 2013, 1-9.

[12] Kurt, B,; Simsek, Y. Frobenius Euler type polynomials related to HermiteBernoulli polynomials. Proceedings of the International Conferences on $\mathrm{Nu}-$ merical Analysis and Applied Mathematics, Amer, Inst. Phys. Conf. Proc. 2011, 1389, 385-388.

[13] Kim, D.S.; Kim, T. A note on polyexponential and unipoly functions. Russ. J. Math. Phys. bf 2019, 26(1), 40-49.

[14] Kim, T.; Kim, D.S. An identity of symmetry for the degenerate FrobeniusEuler polynomials. Math. Slovaca. 2018, 68(1), 239-243.

[15] Kim, T.; Kwon, H.I.; Seo, J.J. On the degenerate Frobenius-Euler polynomials. arXiv:1507.04846v1 [math. N.T] 17 July 2015.

[16] Kim, D.S.; Kim, T.; Seo, J.J. Higher-order Bernoulli, Frobenius-Euler and Euler polynomials, J. Comput. Anal. Appl. 2014, 17(1), 147-155.

[17] Kim, T. A note on degenerate Stirling polynomials of the second kind. Proc. Jangjeon Math. Soc. 2017, 20(3), 319-331.

[18] Kim, T.; Kim, D.S. Degenerate polyexponential functions and degenerate Bell polynomials. J. Math. Anal. Appl. 2020, 487(2), 124017.

[19] Kim, D.S.; Kim, T. A note on a new type of degenerate Bernoulli numbers. Russ. J. Math. Phys. 2020, 27(2), 227-235.

[18] Kim, T Kim, D.S.; Kim, H.-Y.; Jang, L.C. Degenerate poly-Bernoulli numbers and polynomials, Informatica. 3(13) (2020), 2-8.

[19] Kim, D.S.; Kim, T.; Komatsu, T.; Kwon, H.I. Barnes multiple Bernoulli and generalized Barnes multiple Frobenius-Euler mixed-type polynomials. Adv. Difference Equ. 2014, 2014:238.

[20] Ozden, H.; Cangul, I.N.; Simsek, Y. Remarks on $q$-Bernoulli numbers associated with Daehee numbers. Adv. Stud. Contemp. Math. (Kyungshang). 2009, 18(1), 41-48.

[21] Roman, S. The umbral calculus. Pure App. Math. Vol. 111. Academic Press, Inc., New York, 1984.

[22] Ryoo, C.S. A note on the Frobenius-Euler polynomials. Proc. Jangjeon Math. Soc. 2011, 14(4), 495-510.

[23] Simsek, Y. Identities on the Changhee numbers and Apostol-type Daehee polynomial. Adv. Stud. Contemp. Math. (Kyungshang). 2017, 27(2), 199-212.

[24] Simsek, Y. Generating functions for $q$-Apostol type Frobenius-Euler numbers and polynomials. Axioms, 2012, 1, 395-403.

[25] Simsek, Y. Generating functions for generalized Stirlings type numbers array type polynomials, Eulerian type polynomials and their application. Fixed Point Theory and Appl. doi:10.1186/1687-1812-2013-87, 2013. 